\title{
Drogenpolitik: Ja zum Bewährten, kein Konsens beim Neuen
}

Die Bilder vom Zürcher Platzspitz machten Schlagzeilen und gingen um die Welt: schwankende Schwerstsüchtige dort, wo Stadtbewohner auf ihrem morgendlichen Spaziergang mit dem Hund unterwegs waren, gebrauchte Spritzen - Aids war bereits ein Thema - neben Kinderspielplätzen, bettelnde Junkies - wenn auf Entzug, spürbar aggressiv - und eine ideologisierte, zu nichts führende Debatte. Bis sich unter dem Eindruck dieses allgegenwärtigen Elendes allmählich die Einsicht durchsetzte, dass Menschen auch dann ein Anrecht auf medizinische und soziale Unterstützung haben, wenn sie ihre illegalen Drogen nicht aufgeben können.

Ende der 80er Jahre begann eine Entwicklung, deren Resultate nun im Urnengang vom 30. November 2008 ihre rechtliche Grundlage erhalten sollen: die 4-SäulenPolitik mit Prävention, Therapie, Schadensminderung und Repression.

Sie beinhaltet einen breiten Fächer von Massnahmen und Angeboten. So haben leicht erhältliche, saubere Einwegspritzen und das damals heiss diskutierte Angebot der sogenannten «Fixer-Stübli», in denen in Ruhe gefixt werden konnte, die HIV-Neuinfektionsrate positiv beeinflussen können. Niederschwellige medizinische Angebote verbesserten den Gesundheitszustand der Drogensüchtigen. Teilbetreute Wohnprojekte und Arbeitsprojekte führten zu einer besseren sozialen Integration. Betreuung und Therapie haben vielen Menschen wieder eine Perspektive eröffnet.

Es sind eindrucksvolle Zahlen: 1500 stationäre Therapieplätze, 17500 Menschen mit einer Methadonsubstitution, 1500 Schwerstsüchtige in einer heroingestützten und etwa 20000 in ambulanter Behandlung: Alle diese Menschen sind nicht mehr auf der Strasse, sie haben die Möglichkeit, sich sozial zu stabilisieren, Verantwortung für ihr Leben zu übernehmen und einen Weg zurück in die Gesellschaft zu finden.

Die 4-Säulen-Politik ist eine eigentliche Erfolgsstory, die der Schweizer Drogenpolitik - auch international - zu positiven Schlagzeilen verholfen hat. Ein Nein an der Urne wäre ein Rückfall in eine ideologisierte drogenpolitische Diskussion, die niemand ernstlich wollen kann. Wer die 4-Säulen-Politik ablehnt, gibt sich der Illusion einer drogenfreien Gesellschaft hin, die es notfalls mit Repression durchzusetzen gelte. Wir sehen nirgends auf der Welt Anzeichen dafür, dass Repression allein zu Lösungen führt.

Was auch für Cannabis gilt: Repression allein zeigt keine Wirkung! Die Hanfinitiative versucht, einen Weg aus einer verfahrenen Situation zu öffnen. Die etwa 500000 gelegentlichen oder regelmässigen Konsumenten zeigen deutlich, dass sich das Problem nicht polizeilich lösen lässt. Wir brauchen neue Lösungsansätze - einen Schutz der Jugendlichen und eine Entkriminalisierung der Konsumenten, ohne dass die Droge verharmlost wird.

Die Hanfinitiative, über die das Schweizer Stimmvolk ebenfalls am 30. November abstimmt, vermochte die Delegierten der FMH aber nicht zu überzeugen. Sie haben an der Delegiertenversammlung vom 4. September 2008 an der Stimmfreigabe festgehalten. Mit einem klaren Ja zum Betäubungsmittelgesetz sagt die FMH ja zum Bewährten. Dagegen ist die Stimmfreigabe zur Hanfinitiative als Ausdruck eines anhaltenden Unbehagens zu werten. Unbestritten ist, dass die aktuelle Situation nicht befriedigt. Über den Weg aus der Sackgasse besteht aber noch keine Einigung. Noch hat die Diskussion über die Droge Cannabis in der ganzen Gesellschaft einen weiten Weg vor sich - von einer ideologisierten, von Vorurteilen und Ängsten geprägten zu einer faktenbasierten und nüchternen Debatte, die es erlauben wird, konsensfähige und praktikable Lösungen zu entwickeln.

Dr. med. Christine Romann, Mitglied des Zentralvorstands der FMH, Verantwortliche Ressort Gesundheitsförderung und Prävention 